\title{
Micromixing of a Two Phase System in a Stirred Tank with Multiple Impellers
}

\author{
Lei Yang, Jingcai Cheng, Ping Fan, Chao Yang \\ Key Laboratory of Green Process and Engineering, Institute of Process Engineering Chinese Academy of Sciences, Beijing 100190, China \\ Email: jccheng@mail.ipe.ac.cn, chaoyang@home.ipe.ac.cn
}

Received 2012

\begin{abstract}
The competitive iodide/iodate reaction scheme was used to ascertain the micromixing in the stirred solid-liquid systems. Two different glass beads from 450 to $1250 \mu \mathrm{m}$ were tested. The effect of solid particles on reaction selectivity with multiple impellers at different feed points has been investigated. It was confirmed that glass beads as a second phase were suitable for the study. The segregation index has changed significantly only for the medium-sized particles at relatively high solid holdups. The cloud formation was clearly observed for the medium-sized particles at a concentration of $12.12 \mathrm{wt}$. \%. When feeding into the clear liquid above the cloud, the value of the segregation index increased significantly. However, in the presence of particles of 1-1.25 mm, the influence on the selectivity was negligible when the agitation speed was increased.
\end{abstract}

Keywords: Micromixing; Parallel Reactions; Two Phases; Multiple Impellers

\section{Introduction}

Micromixing could have an effect on the selectivity, yield and quality of the desired products in many industrial processes including precipitation, mineral processing, crystallization and biochemical processes. Poor micromixing may reduce the productivity of the desired products and also leads to higher purification costs. Therefore, many physical and chemical methods easy to be implemented have been developed to characterize micromixing. A large amount of work has been done to improve the micromixing in single-phase systems, while relatively little work has been conducted in the two-phase systems especially with multiple impellers ${ }^{[1,2]}$. The dissipation rate of local energy determines the local micromixing. Techniques such as laser Doppler velocimetry (LDV) and particle image velocimetry (PIV) can be used to give reliable values of local energy dissipation in single-phase systems. However, it is much difficult for them to be applied to dense slurries which are commonly opaque. So more experiments need to be undertaken to provide data for multiphase systems. Furthermore, the models used by computational fluid dynamics (CFD) like turbulence model are not so mature. The data are crucial for successful turbulence modelling of multi-phase systems especially for high solids concentration systems.

The influence of suspended solids on the selectivity of fast reactions has been investigated for a few years, but the results are a little contradictory. Villermaux et al. (1994) [1] used the iodide/iodate method to study the effect of suspended solids $(0<$ $\left.d_{\mathrm{p}}<40 \mu \mathrm{m}\right)$ on the micromixing in stirred reactors. They found that the micromixing efficiency was enhanced by the presence of solids without significantly change in the power consumption. However, after Guichardon et al. (1995) [2] calculated the loss of iodine during filtration, the glass beads $\left(20 \mu \mathrm{m}<d_{\mathrm{p}}<\right.$ $1300 \mu \mathrm{m}$ ) had a negligible effect on micromixing up to $5 \mathrm{wt}$ \%. According to Barresi (1997) [3], significant changes in selectiv- ity were observed only at relatively high particle loadings ( $>10$ vol. $\%$ for the glass spheres $\left(d_{\mathrm{p}}=100-177 \mu \mathrm{m}\right.$ and $\left.425-500 \mu \mathrm{m}\right)$ and the selectivity was not affected with larger cylindrical PET beads (equivalent diameter $=3 \mathrm{~mm}$ ). In 1999, Brilman et al. [4] investigated the effect of particles $(70-700 \mu \mathrm{m})$ on product distribution using the diazo-coupling reaction. They found that the segregation index increased while the holdup was enhanced except the particles of $290 \mu \mathrm{m}$. Barresi (2000) [5] reported the effect of particles on reaction selectivity and related it to the changes in the power input and hydrodynamics of the suspension. With the Rushton turbine, a lower selectivity was observed in the slurry. On the contrary, selectivities were higher in the slurry than those in the single phase when the pitched blade was used. Recently, micromixing was unaffected near the impeller and near the surface with glass beads of $500 \mu \mathrm{m}$ at concentrations up to $2.5 \mathrm{wt}$.\% by Hofinger et al. [6]. Cloud formation was observed, and when feeding into the clear liquid above the cloud, the segregation index increased significantly.

From the literature review above, it can be concluded that there still exists disagreement between researchers on the effect of solid particles. The aim of this paper is to investigate the effect of solid particles on micromixing and give data on mean energy dissipation with multiple stirrers in the solid-liquid systems.

\section{Experimental Set-Up and Methods}

\subsection{Experimental Setup and Agitation Condition}

Experiments have been carried out at room temperature in a cylindrical Perspex vessel (diameter, $T=0.384 \mathrm{~m}$ ) with a dished bottom. A schematic of the experimental setup is given in Figure 1. The tank is equipped with four baffles of width $T / 10$ perpendicularly. In this work, a Rushton turbine (blade: height $21 \mathrm{~mm}$, width $32 \mathrm{~mm}$, thickness $1 \mathrm{~mm}$ ) and a $45^{\circ}$ down pump- 
ing 6-blade pitched blade turbine (blade: height $25 \mathrm{~mm}$, width $47 \mathrm{~mm}$, thickness $1 \mathrm{~mm}$ ), all of which have a diameter $D=T / 3$, were used. In order to avoid surface aeration, $H / T$ is 1.6 . The impeller off-bottom clearance is $T / 3$. The power drawn by the impeller was determined via the torque from a strain gauge attached to the shaft. A steel pipe with an inner diameter of 2 $\mathrm{mm}$ was used at each position. The geometrical details of the feed pipe tip are given in Table 1. Different sizes of glass beads were tested:

1) medium size: $d_{\mathrm{p}}=600-425 \mu \mathrm{m}\left(\rho_{s}=2.431 \mathrm{~g} / \mathrm{L}\right)$;

2) large size: $d_{\mathrm{p}}=1.25 \mathrm{~mm}>d>1 \mathrm{~mm}\left(\rho_{s}=2.431 \mathrm{~g} / \mathrm{L}\right)$.

\subsection{Chemical Test Reaction}

Micromixing experiments were carried out in the semi-batch mode using the reaction developed by Fournier et al. [7]. According to the following steps:

$$
\begin{gathered}
\mathrm{H}_{2} \mathrm{BO}_{3}^{-}+\mathrm{H}^{+} \rightarrow \mathrm{H}_{3} \mathrm{BO}_{3} \\
5 \mathrm{I}^{-}+\mathrm{IO}_{3}^{-}+6 \mathrm{H}^{+} \leftrightarrow 3 \mathrm{I}_{2}+3 \mathrm{H}_{2} \mathrm{O} \\
\mathrm{I}_{2}+\mathrm{I}^{-} \leftrightarrow \mathrm{I}_{3}^{-}
\end{gathered}
$$

The equilibrium constant $K_{B}$ of (iii) is well known as a function of the temperature. [8]

$$
\log _{10} K_{B}=555 / T+7.355-2.575 \log _{10} T, \quad K_{B} \text { in } \mathrm{M}^{-1}
$$

The experiment procedure consists of injecting $0.03 \mathrm{~L}$ of sulphuric acid $\left(\left[\mathrm{H}^{+}\right]=1.0 \mathrm{M}\right)$ to the solution containing iodide, iodate and borate ions, whose concentrations follow that of Guichardon and Falk [9]. It has been confirmed that $X_{\mathrm{Q}}$, the selectivity of iodine formation, whose value lies between 0 and 1 , is a measurement of micromixing efficiency. $X_{\mathrm{Q}}$ is defined as: $X_{\mathrm{Q}}=Y / Y_{S T}$, where,

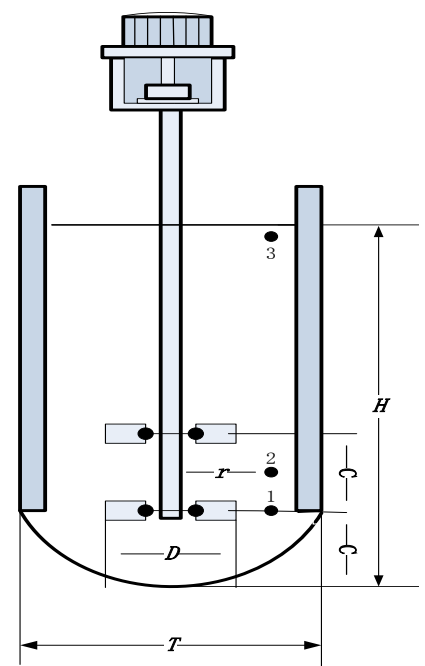

Figure 1. Schematic of the tank and feed points. Table 1. Geometrical details of the feed pipe tip.

\begin{tabular}{cccc}
\hline \multirow{2}{*}{$\begin{array}{c}\text { Feed } \\
\text { position }\end{array}$} & Position $<1>$ & Geometrical details \\
\cline { 2 - 4 } & 2.2 & Position $<2>$ & Position $<3>$ \\
\hline $2 r / D$ & 0.21 & 0.31 & 2.2 \\
z/H & & & 0.84 \\
\hline
\end{tabular}

$$
Y=\frac{2\left(n_{\mathrm{I}_{2}}+n_{\mathrm{I}_{3}^{-}}\right)}{n_{\mathrm{H}_{0}^{+}}}=\frac{2 V_{\text {tank }}\left(\left[\mathrm{I}_{2}\right]+\left[\mathrm{I}_{3}^{-}\right]\right)}{V_{\text {injection }}\left[\mathrm{H}^{+}\right]_{0}}
$$

and

$$
Y_{\mathrm{ST}}=\frac{6\left[\mathrm{IO}_{3}^{-}\right]_{0}}{6\left[\mathrm{IO}_{3}^{-}\right]_{0}+\left[\mathrm{H}_{2} \mathrm{BO}_{3}^{-}\right]_{0}}
$$

Based on mass balances on iodine atoms, the following expression is produced:

$$
\left[\mathrm{I}^{-}\right]=\left[\mathrm{I}^{-}\right]_{0}-5 / 3\left(\left[\mathrm{I}_{2}\right]+\left[\mathrm{I}_{3}^{-}\right]\right)-\left[\mathrm{I}_{3}^{-}\right]
$$

The value of XQ is calculated by combining (1) and (4).

\section{Results and Discussion}

\subsection{Determination of Molar Extinction Coefficient}

The molar extinction coefficient, $\varepsilon$, of triiodide ion was determined by measuring the optical density of the solutions containing potassium iodide and iodine with a ratio of 2:1. The results are depicted in Figure 2. In this paper, the analysis was conducted at $353 \mathrm{~nm}$ with a single beam where the interference with iodide ion and solvent absorption could be reduced. At $353 \mathrm{~nm}$, we found that $\varepsilon$ was $2624 \mathrm{~mol}^{-1} \mathrm{~m}^{2}$, which is in good agreement with the literature $[7,10]$.

\subsection{The Influence of Feed Time}

With the purpose of finding out the conditions in which micromixing were free from macromixing, we changed the feed rate both in the single phase and the slurry system. From Figure 3, it can be seen that the segregation indexes come to asymptotic values when the feed time is larger than $2145 \mathrm{~s}$, no matter what the system is. Thus, all our experiments were carried out with feed times larger than $2145 \mathrm{~s}$.

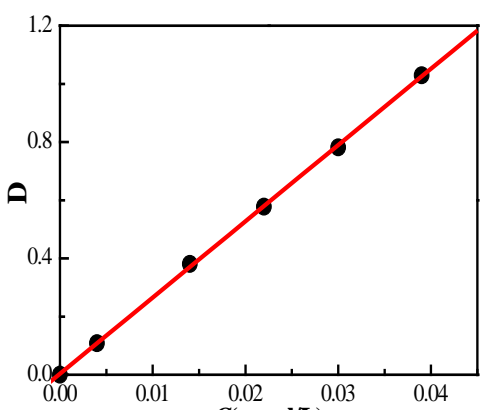

Figure 2. The molar extinction coefficient.

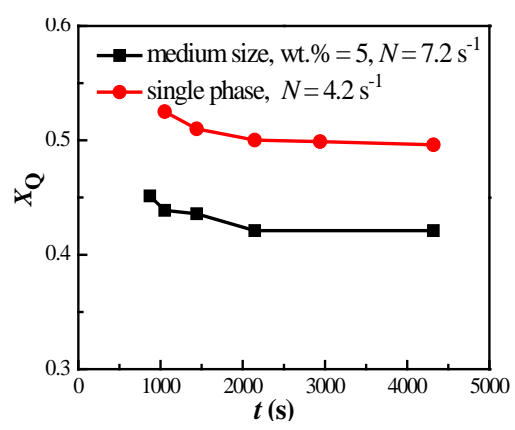

Figure 3. Influence of feed time on segregation index, feed point 3. 


\subsection{Preliminary Studies}

The absorption of iodine onto the solids surface in the liquid phase has been reported in previous studies. Thus, preliminary tests were undertaken to check whether there was any absorption. To verify the stability, experiments were carried out in single phase and the suspension. As is described in Figure 4, samples were taken out of the tank in 20 minutes after the injection in all of our experiments. But the deviations are no more than $\pm 2 \%$. So the adsorption could be negligible.

\subsection{Effects of Agitation Speed and Feed Position}

Agitation speed and feed position play important roles in solid suspension. The just-suspended speed $N_{\mathrm{js}}$ was estimated following Zwietering's method [11]. Experiments were performed at a speed above $N_{\mathrm{js}}$. Figure 5 shows $X_{\mathrm{Q}}$ values for 5 agitation speeds at 2 feed points and 2 solid concentrations. As for all the conditions considered, the higher the stirrer speed which can improve turbulence, the higher the value of $X_{\mathrm{Q}}$. By combining single phase and medium-sized particle suspension with a mass fraction of $5 \%$, it is revealed that the feeding at point 2 is better in our experimental conditions. For the medium-sized particles, when the amount of solids increases, the segregation index is enhanced. By comparing single phase and the suspension at feed point 2, it can be easily concluded that the segregation index is augmented by the enlarged particle size.

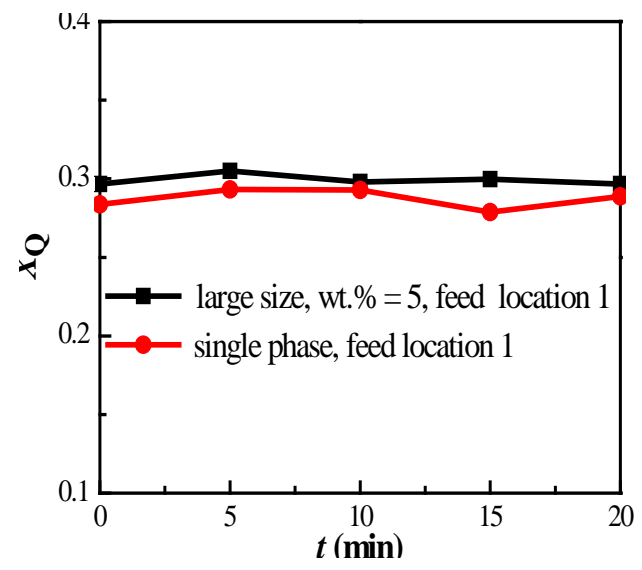

Figure 4. The test for absorption onto the solid particles, $N=9.5 \mathrm{~s}^{-1}$.

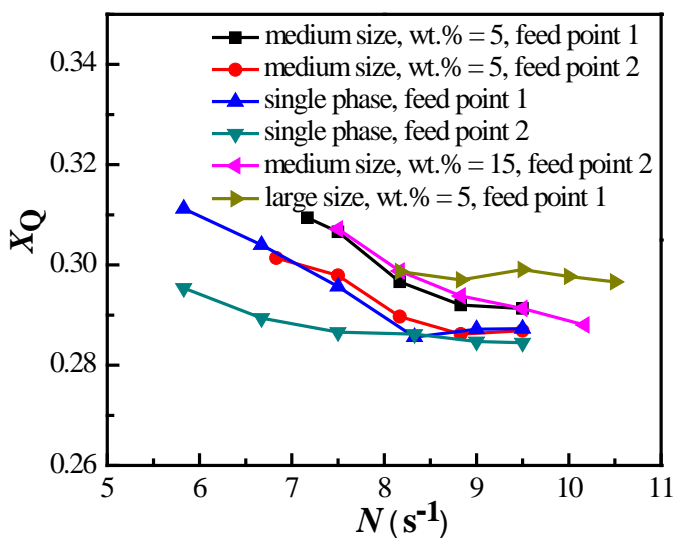

Figure 5. Effects of impeller speed, feed point on $X_{Q}$.

\subsection{Mean Energy Dissipation and Solid Loading}

Figure 6 describes the segregation index as a function of solid loading of different sized particles. During the experiments, it was at 12.12 wt. \% when the cloud formation was clearly observed for the particles with medium size. When feeding into the clear liquid above the cloud, the value of $X_{\mathrm{Q}}$ increased significantly (closely similar to [12]). As is depicted in Figure 6(b), the enhancement in the segregation index is examined but does not increase very notably with the solid concentration ranging from 0 to 20 by weight. When particle concentration is less than 5 wt. \%, the influence is found to be negligible, which is in agreement with that of Barresi (1997). Probably the reason is that the impeller speed used is high enough to achieve a good mixing effect. In contrast, the segregation index almost keeps constant though the solid loading is improved for the largesized system (Figure 6(a)). The results of this study are in accordance with those presented by Guichardon et al. (1995), who found a negligible influence of solid particles $\left(\rho_{s}=2500 \mathrm{~kg} / \mathrm{m}^{3}\right.$, $d_{\mathrm{p}}=1250 \mu \mathrm{m}$ ) on the segregation index.

As is well-recognised, the power input in a stirred tank is mostly consumed in the impeller region and especially in the discharge stream. Considering our experimental results, mean energy dissipation was decreased while the particle size increased at the same holdup. This phenomenon has been explained by many researchers [13-15]. As suggested by Brilman et al. (1999), the increase of segregation index may be caused by: (1) more energy was dissipated in particle-particle collisions and particle-wall collisions; (2) the amount of stagnant

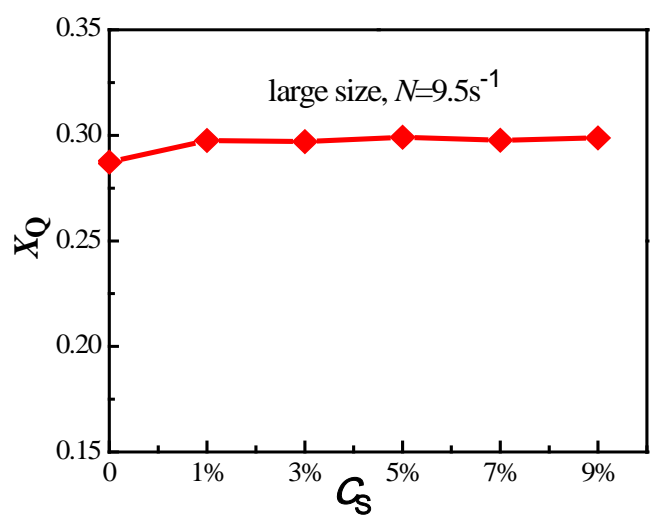

(a)

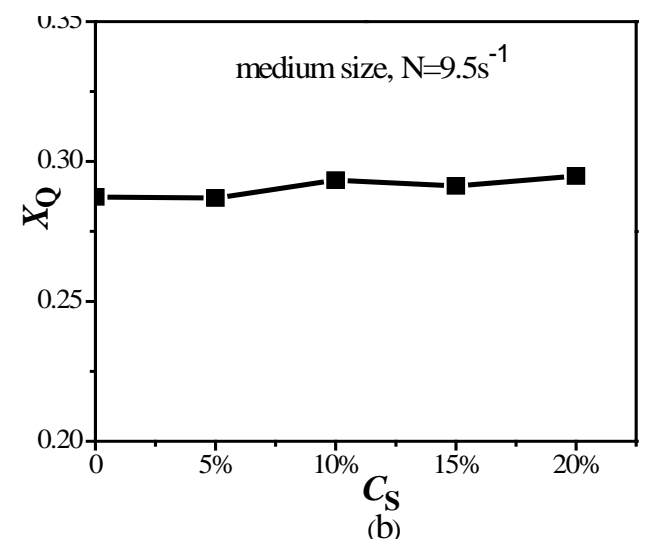

Figure 6. Effect of solid loading at feed location 1. 


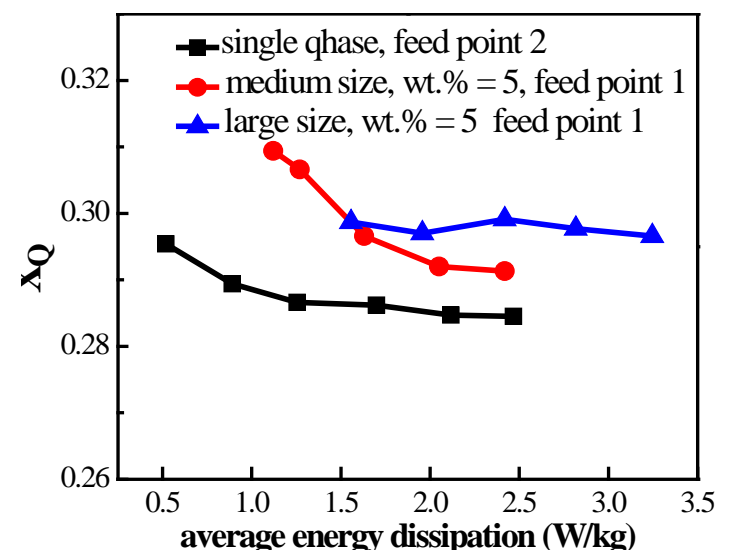

Figure 7. Comparison of mean specific energy dissipations.

liquid moving with the particles was increased. Figure 7 describes the correlation between the mean energy dissipation and the segregation index. At higher particle sizes, there is an increase in segregation index due to more energy distributed to the particles. The results accord with those of Barresi (2000) and Brilman et al. (1999).

\section{Conclusions}

The effect of solid particles on reaction selectivity in a dishedbottom stirred tank with multiple impellers at different feed points has been investigated. It has been confirmed that glass beads as a second phase were suitable for the study. Large changes in the segregation index were obtained only for the medium-sized particles at relatively high solid holdups. During the experiments, the cloud formation was clearly observed for the medium-sized particles at the hold of 12.12 wt. \%. When feeding into the clear liquid above the cloud, the value of $X_{\mathrm{Q}}$ increased significantly and was up to 0.56 . In the presence of the glass particles of $1-1.25 \mathrm{~mm}$, the influence on the selectivity was negligible though the solid concentration was enhanced when feeding at the tip of the pitched blade turbine.

From our experimental results, it can be concluded that the segregation index improved but not apparently in the feed points studied when the agitation speeds increased. In order to reduce the selectivity towards undesired product, it is indispensable to improve the mixing, such as by increasing the circulation. However, it may consume more power. As suggested by Bourne and Hilber [16], an alternative could be the use of multiple feeds. Further work has been intended to find out the maximum energy dissipation rate of the pitched blade turbine and then carry out multiple feeds.

\section{Acknowledgements}

The authors acknowledge the financial support from the 973 Program (2012CB224806), the National Natural Science Fund for Distinguished Young Scholars (21025627), the National Natural Science Foundation of China (21106154, 20990224),
863 Project (2012AA061503), Beijing Natural Science Foundation (2112038) and Jiangsu Province Project (BY2009133).

\section{REFERENCES}

[1] J. Villermaux, L. Falk, and M. C. Founier, "Potential use of a new parallel reaction system to characterize micromixing in stirred reactors,” AIChE Symp. Ser., vol. 90, pp. 50-54, 1994.

[2] P. Guichardon, L. Falk, M. C. Founier, and J. Villermaux, "Study of micromixing in a liquid-solid suspension in a stirred tank,” AIChE Symp. Ser., vol. 91, pp. 123-130, 1995.

[3] A. A. Barresi, "Experimental investigation of interation between turbulent liquid flow and solid particles and its effects on fast reactions,” Chem.Eng. Sci., vol. 52, pp. 807-814, 1997.

[4] D. W. F. Brilman, R. Antink, W. P. M. van Swaaij, and G. F. Versteeg, "Experimental study of the effect of bubbles, drops and particles on the product distribution for a mixing sensitive, parallel-consecutive reaction system,” Chem. Eng. Sci., vol. 54, pp. 2325 - 2337, 1999.

[5] A. A. Barresi, "Selectivity of mixing-sensitive reactions in slurry systems,” Chem.Eng. Sci., vol. 55, pp. 1929-1933, 2000.

[6] J. Hofinger, R. W. Shape, W. Bujalski, S. Bakalis, M. Assirelli, A. Eaglesham, and A. W. Nienow, "Micromixing in two-phase (G-L and S-L) systems in a stirred vessel,” Can. J. Chem. Eng., vol. 89, pp. 1 - 11, 2011.

[7] M. C. Fournier, L. Falk, and J. Villermaux, “A new parallel competing reaction system for assessing micromixing efficiency— Experimental approach," Chem.Eng. Sci., vol. 51, pp. 5053-5064, 1996.

[8] D. A. Palmer, R. W. Ramette, and R. E. Mesmer, "Triiodide ion formation equilibrium and activity coefficients in aqueous solution,” J. Solution Chem., vol. 13, pp. 673-683, 1984.

[9] P. Guichardon and L. Falk, "Characterisation of micromixing efficiency by the iodide-iodate reaction system. Part I: experimental procedure,” Chem. Eng. Sci.,vol. 55, pp. 4233 - 4243, 2000.

[10] Awtrey and Connick, "The absorption spectra of $\mathrm{I}_{2}, \mathrm{I}_{3}^{-}, \mathrm{IO}_{3}^{-}$, $\mathrm{S}_{2} \mathrm{O}_{6}^{2-}, \mathrm{S}_{2} \mathrm{O}_{3}^{2-}$. Heat of the Reaction $\mathrm{I}_{3}^{-}=\mathrm{I}_{2}+\mathrm{I}^{-}$," J. Am. Chem. Soc, vol. 73, pp. 1844-1843, 1951.

[11] Zwietering, Th. N, "Suspension of Solid Particles in Liquid by Agitators,” Chem. Eng. Sci., vol. 8, pp. 244-253, 1958.

[12] M. Assirelli, W. Bujalski, A. Eaglesham, and A. W. Nienow, "Study of micromixing in a stirred tank using a Rushton Turbine: Comparison of feed positions and other mixing devices," Chem. Eng. Res. Des., vol. 80, pp. 855 - 863, 2002.

[13] Yu. A. Buyevich, "Fluid dynamics of coarse dispersions,” Chem. Eng. Sci. vol. 49, pp. 1217-1228, 1994.

[14] J. O. Hinze, "Turbulent fluid and particle interaction,” In Proceedings of the International Symposium Two-Phase Systems, eds G. Hetsroni, S. Sideman and J. P. Hartnett Prog, Heat Mass Transfer, vol. 6, pp. 433-452. Pergamon Press, Oxford, 1972.

[15] S. Elgobashi, “On predicting particle-laden turbulent flows,” Appl. Sci. Res., vol. 52, pp. 309-329, 1994.

[16] J. R. Bourne and C. P. Hilber, "The productivity of micromixing controlled reactions: effect of feed distribution in stirred tanks," Chem. Eng. Res. Des. vol. 68A, pp. 51-56, 1990. 